\title{
Qualitative Analysis on Differential, Fractional Differential, and Dynamic Equations and Related Topics
}

\author{
Said R. Grace, ${ }^{1}$ Taher S. Hassan, ${ }^{2,3}$ Shurong Sun, ${ }^{4}$ and Elvan Akin ${ }^{5}$ \\ ${ }^{1}$ Department of Engineering Mathematics, Faculty of Engineering, Cairo University, Giza, Egypt \\ ${ }^{2}$ Department of Mathematics, Faculty of Science, University of Hail, Hail 2440, Saudi Arabia \\ ${ }^{3}$ Department of Mathematics, Faculty of Science, Mansoura University, Mansoura 35516, Egypt \\ ${ }^{4}$ School of Mathematical Sciences, University of Jinan, Jinan, Shandong 250022, China \\ ${ }^{5}$ Missouri University of Science Technology, 310 Rolla Building, Rolla, MO 65409-0020, USA
}

Correspondence should be addressed to Said R. Grace; saidgrace@yahoo.com

Received 11 July 2016; Accepted 11 July 2016

Copyright (C) 2016 Said R. Grace et al. This is an open access article distributed under the Creative Commons Attribution License, which permits unrestricted use, distribution, and reproduction in any medium, provided the original work is properly cited.

This issue on qualitative analysis on differential, fractional differential, and dynamic equations and related topics aims at an all-around research and the state-of-the-art theoretical, numerical, and practical achievements that contribute to this field. This issue contains the following features.

Oscillation and Asymptotic Behavior. S. R. Grace and E. Akin investigate the asymptotic behavior of nonoscillatory solutions of certain forced integrodifferential equations. From the obtained results, they drive a technique which can be applied to some related integrodifferential as well as integral equations.

T. S. Hassan and S. R. Grace consider the higher-order functional dynamic equations with mixed nonlinearities and study their oscillatory behavior via comparison with some equations whose oscillatory characters are known and studied extensively in the literature.

Stochastic Delay Differential Equations, Dynamics of Stochastic Coral Reefs Model, Stochastic Predator-Prey System Subject to Lévy Jumps, and Stochastic Resonance in a Multistable System Driven by Gaussian Noise. H. Yuan et al. introduced and analyzed split-step theta (SST) method for nonlinear neutral stochastic differential delay equations (NSDDEs). The asymptotic mean square stability of the split-step theta (SST) method is considered for nonlinear neutral stochastic differential equations. It is proved that, under the one-sided
Lipschitz condition and the linear growth condition, for all positive step sizes, the split-step theta method with $\theta \in$ $(1 / 2,1]$ is asymptotically mean square stable. The stability for the method with $\theta \in[0,1 / 2]$ is also obtained under a stronger assumption. It further studies the mean square dissipativity of the split-step theta method with $\theta \in(1 / 2,1]$ and proves that the method possesses a bounded absorbing set in mean square independent of initial data.

Z. Huang work is devoted to discerning asymptotic behavior dynamics through the stochastic coral reefs model with multiplicative nonlinear noise. By support theorem and Hörmander theorem, the Markov semigroup corresponding to the solutions is to prove the Foguel alternative. Based on boundary distributions theory, the required conservative operators related to the solutions are further established to ensure the existence of a stationary distribution. Meanwhile, the density of the distribution of the solutions either converges to a stationary density or weakly converges to some probability measure.

$\mathrm{X}$. Wang and X. Meng investigate a new nonautonomous impulsive stochastic predator-prey system with the omnivorous predator. First, they show that the system has a unique global positive solution for any given initial positive value. Second, the extinction of the system under some appropriate conditions is explored. In addition, they obtain the sufficient conditions for the almost sure permanence in mean and stochastic permanence of the system by using the theory 
of impulsive stochastic differential equations. Finally, they discuss the biological implications of the main results and show that the large noise can make the system go extinct. Simulations are also carried out to illustrate our theoretical analysis conclusions.

P. Shi et al. investigated stochastic resonance (SR) in a multistable system driven by Gaussian white noise. Using adiabatic elimination theory and three-state theory, the signalto-noise ratio (SNR) is derived. They find the effects of the noise intensity and the resonance system parameters $b, c$, and $\mathrm{d}$ on the SNR; the results show that SNR is a nonmonotonic function of the noise intensity; therefore, a multistable SR is found in this system, and the value of the peak changes with changing the system parameters.

Hamiltonian Systems and Dynamic Optimization. F. Pierret and D. F. M. Torres derive the Helmholtz theorem for nondifferentiable Hamiltonian systems in the framework of Cresson's quantum calculus. Precisely, they give a theorem characterizing nondifferentiable equations, admitting a Hamiltonian formulation. Moreover, in the affirmative case, they give the associated Hamiltonian.

D.-S. Wang et al. investigate the effects of terms-oftrade shocks on the spending and current account where households with modified Becker-Mulligan endogenous time preference maximize their utility over an infinite planning period. The results reveal the view that with an endogenous rate of time preference the stability requirements preclude the Harberger-Laursen-Metzler effect in an infinite horizon model. Different from Obstfeld (1982), where households with Uzawa endogenous time preference are considered, deterioration in terms of trade leads to a current increase in expenditure in order to catch the new optimum. These theoretical results are consistent with the empirical evidence by numerical simulations.

Singularly Perturbed Systems and Exponential Attractor for the Boussinesq Equation. $\mathrm{H}$. Xu and Y. Jin investigate a class of semilinear singularly perturbed systems with contrast structures discussed. Firstly, they verify the existence of heteroclinic orbits connecting two equilibrium points about the associated systems for contrast structures in the corresponding phase space. Secondly, the asymptotic solutions of the contrast structures by the method of boundary layer functions and smooth connection are constructed. Finally, the uniform validity of the asymptotic expansion is defined and the existence of the smooth solutions is proved. Singularly perturbed problems are often used as the models of ecology and epidemiology.

F. Geng et al. studied the existence of exponential attractor for the Boussinesq equation with strong damping and clamped boundary condition. The main result is concerned with nonlinearities with supercritical growth. In that case, they construct a bounded absorbing set with further regularity and obtain quasi-stability estimates. Then, the exponential attractor is established in natural energy space.

Bifurcation. N. Wang et al. study a predator-prey model mathematically and numerically. The aim is to explore how some key factors influence dynamic evolutionary mechanism of steady conversion and bifurcation behavior in predatorprey model. The theoretical works have been pursuing the investigation of the existence and stability of the equilibria, as well as the occurrence of bifurcation behaviors (transcritical bifurcation, saddle-node bifurcation, and Hopf bifurcation), which can deduce a standard parameter controlled relationship and in turn provide a theoretical basis for the numerical simulation. Numerical analysis ensures reliability of the theoretical results and illustrates that three stable equilibria will arise simultaneously in the model. It testifies the existence of Bogdanov-Takens bifurcation, too. It should also be stressed that the dynamic evolutionary mechanism of steady conversion and bifurcation behavior mainly depend on a specific key parameter. In a word, all these results are expected to be of use in the study of the dynamic complexity of ecosystems.

Said R. Grace

Taher S. Hassan Shurong Sun

Elvan Akin 


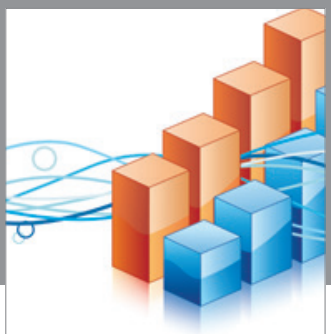

Advances in

Operations Research

vatem alat4

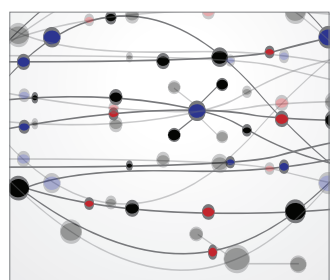

\section{The Scientific} World Journal
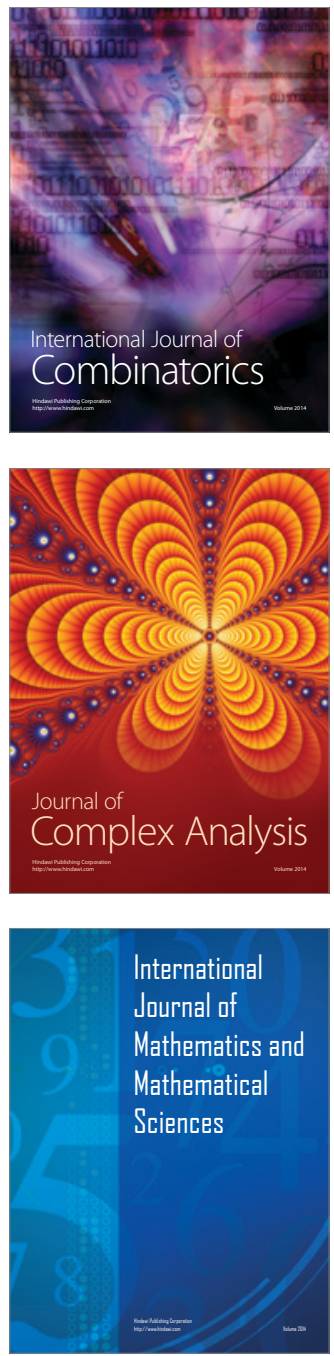
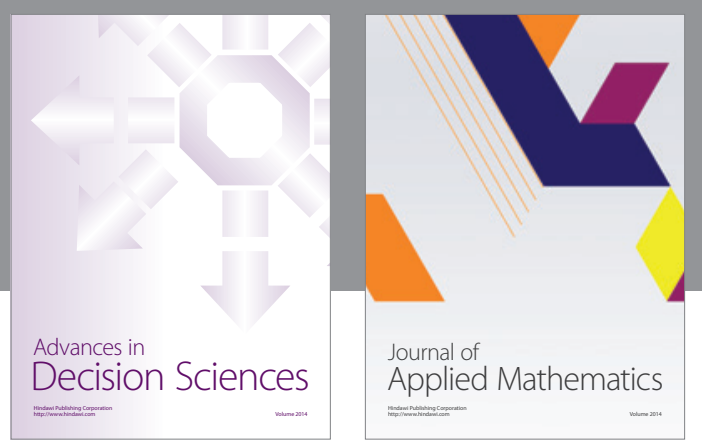

Algebra

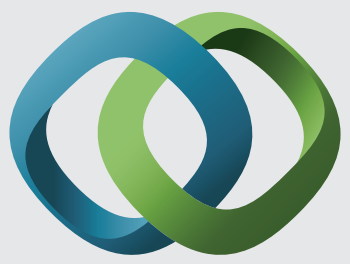

\section{Hindawi}

Submit your manuscripts at

http://www.hindawi.com
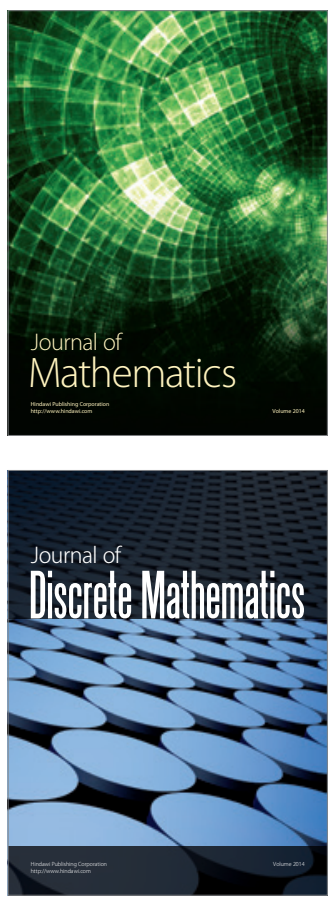

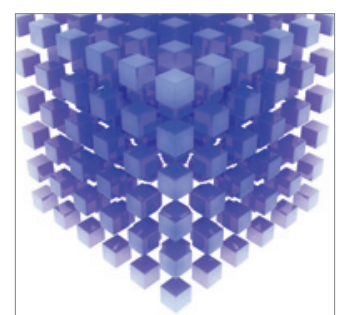

Mathematical Problems in Engineering
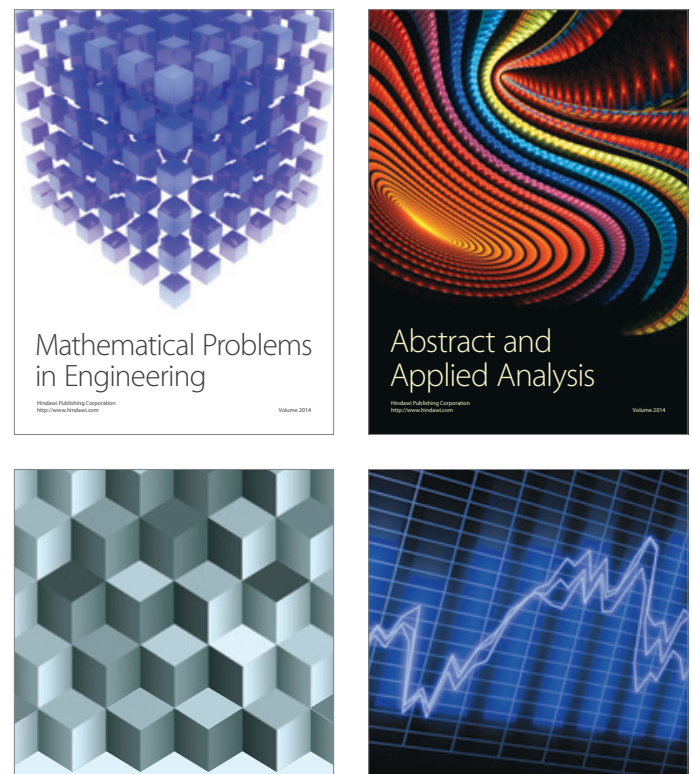

Journal of

Function Spaces

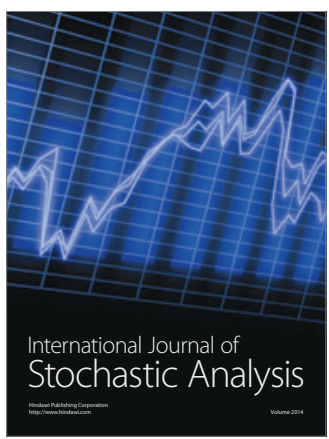

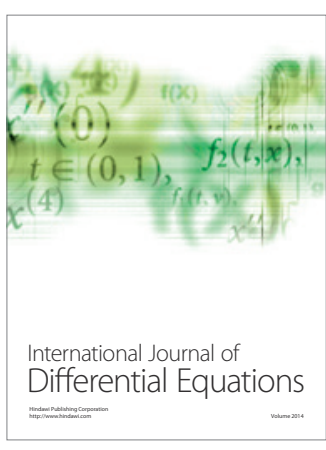
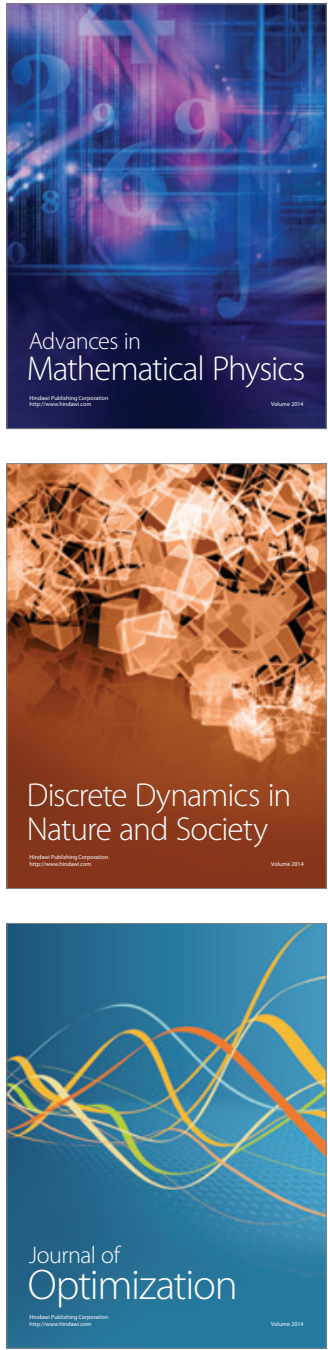